\title{
Latissimus dorsi muscle flap transfer through endoscopic approach combined with the implant after tissue expansion for breast reconstruction of mastectomy patients
}

\author{
Jian-Xun Ma, Bi Li*, You-Chen Xia, Wei-Tao You, Jie Zhang, Yi-Mou Sun, Xu Chang and Yue Lang
}

\begin{abstract}
Background: Implant-based breast reconstruction is easy to be performed but has flaws that an unnatural appearance might be presented when no sufficient coverage existing. While autologous tissue reconstruction also has disadvantages like donor site scar and skin patch effect. There is a demand for a new method to obtain natural and aesthetic appearance while surmounting drawbacks of conventional breast reconstruction surgery.

Methods: A retrospective review of thirty-one patients undergoing tissue expander (TE)/implant two-stage breast reconstruction with latissimus dorsi muscle flap (LDMF) transfer through endoscopic approach in Peking University Third Hospital from April 2016 to August 2020 was performed. The LDMF harvest time, drain time, and complications were reviewed. The 3D volume was obtained to assess the volume symmetry of bilateral breasts. The BREAST-Q reconstruction module was used to evaluate the satisfaction.

Results: The mean endoscopic LDMF harvest time was $90.4 \mathrm{~min}$. In the mean follow-up of 11.2 months, there were no severe capsular contracture happened. The reconstructed side achieved good volume symmetry to the contralateral side $(P=0.256)$. Based on the evaluation of the BREAST-Q scores, the outcome of Satisfaction with Breasts was excellent or good in $87.1 \%$ of the cases.

Conclusions: The novel type of two-stage breast reconstruction protocol, which includes tissue expansion followed by implant insertion with endoscopy-assisted LDMF transfer, could effectively reduce visible scars, avoid the patch effect, while require short time for LDMF harvest and present low incidence of complications. It is a promising method for breast reconstruction because it achieves good outcomes in the mastectomy patients.
\end{abstract}

Keywords: Endoscopic technique, Latissimus dorsi muscle, Implant, Two-stage, Breast reconstruction

\section{*Correspondence: libi0377@126.com}

Department of Plastic Surgery, Peking University Third Hospital, \#49, North Garden Road Haidian District, Beijing 100191, People's Republic of China

\section{Background}

The number of patients suffering from breast cancer is increasing in China [1]. The deficiency of breasts by mastectomy greatly influences women's normal lives, thus the surgery of breast reconstruction is now much in demand.

There are various methods of breast reconstruction for choice. All the procedures are used to supply the 
excessive skin envelop and compensate for the breast tissue loss. As for implant-based reconstruction, there are often criticisms that no sufficient soft tissue coverage on the prosthesis, especially after tissue expansion, cause an unnatural appearance [2]. As for autologous tissue reconstruction, the latissimus dorsi myocutaneous flap has been a workhorse of reconstructive surgery, but it is always used for the reconstruction of small-tomedium breast [3, 4]. Moreover, the conventional harvest technique requires an obvious back incision that can be between 15 and $45 \mathrm{~cm}$ in length, in addition to an axillary incision for pedicle dissection or flap transfer. In addition, there is a trend that patients do not favour a skin patch on the surface of reconstructed breast. Nowadays, endoscopy-assisted techniques are being applied throughout plastic surgery, which makes it possible to harvest the latissimus dorsi muscle flap (LDMF) through a minimal invasive approach.

In consideration of overcoming those drawbacks of sole prosthesis and latissimus dorsi myocutaneous flap breast reconstruction, we innovatively combine endoscopic LDMF transfer with tissue expander (TE) /implant twostage breast reconstruction. Through this new type of breast reconstruction protocol, the long prominent scar on the back could be avoided, and the coverage over the prosthesis could also be enhanced. The purpose of this study is to investigate the aesthetic outcomes in a single surgical group practice and to evaluate its safety and effectiveness.

\section{Methods \\ Patients}

The patients undergoing TE/implant two-stage breast reconstruction in our department from April 2016 to August 2020, in which endoscopy-assisted LDMF transfer was conducted on the second stage, were included in this study. The inclusion criteria for patient selection are defined as (1) female $\geq 20$ years old and $\leq 55$ years old; (2) patients underwent unilateral mastectomy because of breast cancer; (3) existence of LDM and thoracodorsal artery confirmed by ultrasound. The exclusion criteria are defined as (1) poor function of ipsilateral LDM; (2) the contralateral breast has severe ptosis; (3) other contraindications for LDMF or implant surgery.

This study was approved by Peking University Third Hospital Medical Science Research Ethics Committee (No. M2018278). Informed consent was obtained from all patients. All methods performed in this study were in accordance with the Declaration of Helsinki.

\section{Surgical technique}

On the first stage of breast reconstruction, the pectoralis major muscle and the serratus anterior muscle near the anterior axillary line are elevated from the chest wall via the mastectomy incision. Then a $400 \mathrm{ml}$ round TE (Guangzhou Wanhe Plastic Materials Co., Ltd., Guangzhou, China) is inserted into the sub-pectoral and subserratus pocket followed by setting a closed suction drainage (Suzhou Kebang Polymer Medical Apparatus Co., Ltd., Suzhou, China) in the pocket, and the edges between pectoralis major muscle and serratus muscle are sutured together to close the pocket. The volume of saline injected into the TE is approximately $25 \%$ of the total volume of the expander, unless the mastectomy flaps or the muscle pocket do not look healthy or strong enough to tolerate this. The skin incision is closed at last.

One week after operation, the expansion could begin if no signs of ischemia and necrosis of flaps are observed. The volume of water injection is usually $10 \%$ of the expander capacity with interval of one week between inflations. The expansion finishes when the volume of the affected side compared to the contralateral side reaches about $160 \%$ [5].

On the second stage, when the tissue expansion is finished, markings are prepared preoperatively while the patient is in an upright position. The markings include the midline, inframammary fold, lateral edge of breast tissue on the chest wall, and the lateral margin of the LDM along the posterior axillary line, inferior margin at the iliac crest, medial margin along the paravertebral origin, superior margin at the tip of the scapula, and also the incisions (Fig. 1).

During the operation, the patient is placed in a lateral decubitus position with the relevant upper extremity abducted and rested on a supporter. The previous mastectomy incision extending to the posterior axillary fold is opened through the skin. The TE is identified and removed. In the meanwhile, the superior anterior border of LDM is exposed as surgeons continue the macroscopic subcutaneous and submuscular dissection to create an initial optic cavity, which serves as a landmark for subsequent endoscopic muscle dissection. The tumescent solution including $250 \mathrm{ml}$ Lactated Ringer, $1 \mathrm{mg}$ adrenaline, and $200 \mathrm{mg}$ lidocaine, is injected into both subcutaneous and submuscular planes. Then a 10 -mm-diameter rigid endoscope with a 30-degree-angle lens (KARL STORZ Gmbh \& Co. KG, Germany) is set up together with a U-shaped retractor (Shanghai Zhonghetiangong Medical Instrument Co., Ltd., Shanghai, China). The retractor and endoscope inserted through the incision are held by an assistant to maintain an optical cavity that facilitates the dissection (Fig. 2). The retractor which has holes in the tip is connected with vacuum suction in order to suck out the cautery smoke and heat. The subcutaneous plane is dissected first by a long monopolar electrosurgical hook (Shanghai Zhonghetiangong Medical Instrument 


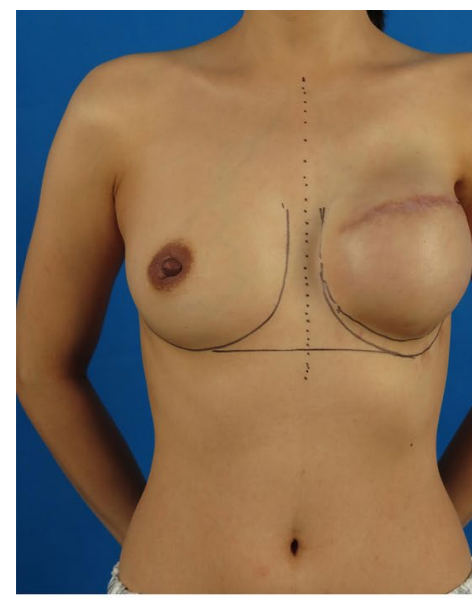

A

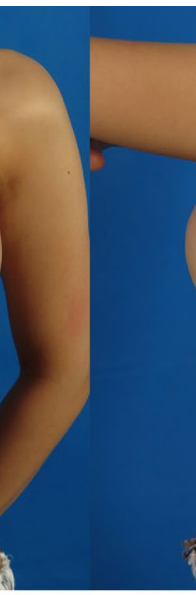

htmona
B

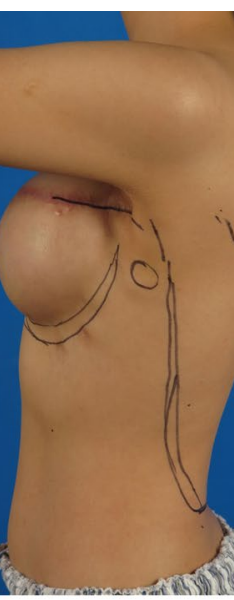

(a)

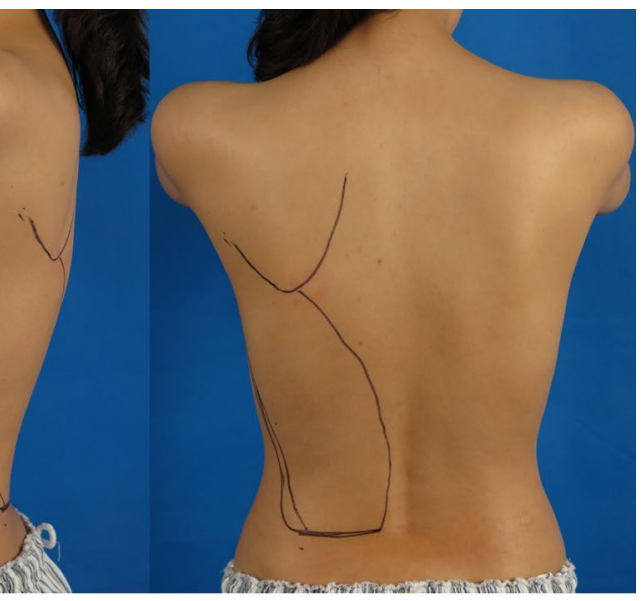

C

Fig. 1 Marking before the endoscopic LDMF transfer combined with implant insertion on the second stage of breast reconstruction. A The midline, borders of bilateral breasts, and inframammary fold are marked on the chest wall. B The mastectomy incision extending to the posterior axillary fold is marked as the endoscopic approach. $\mathbf{C}$ The borders of the latissimus dorsi muscle are marked on the back: the anterior border can be palpated during active muscle contraction, the superior border is marked from the tendinous insertion along the tip of the scapula to the medial border along the paravertebral origin, and the inferior margin is marked at the iliac crest. LDMF latissimus dorsi muscle flap

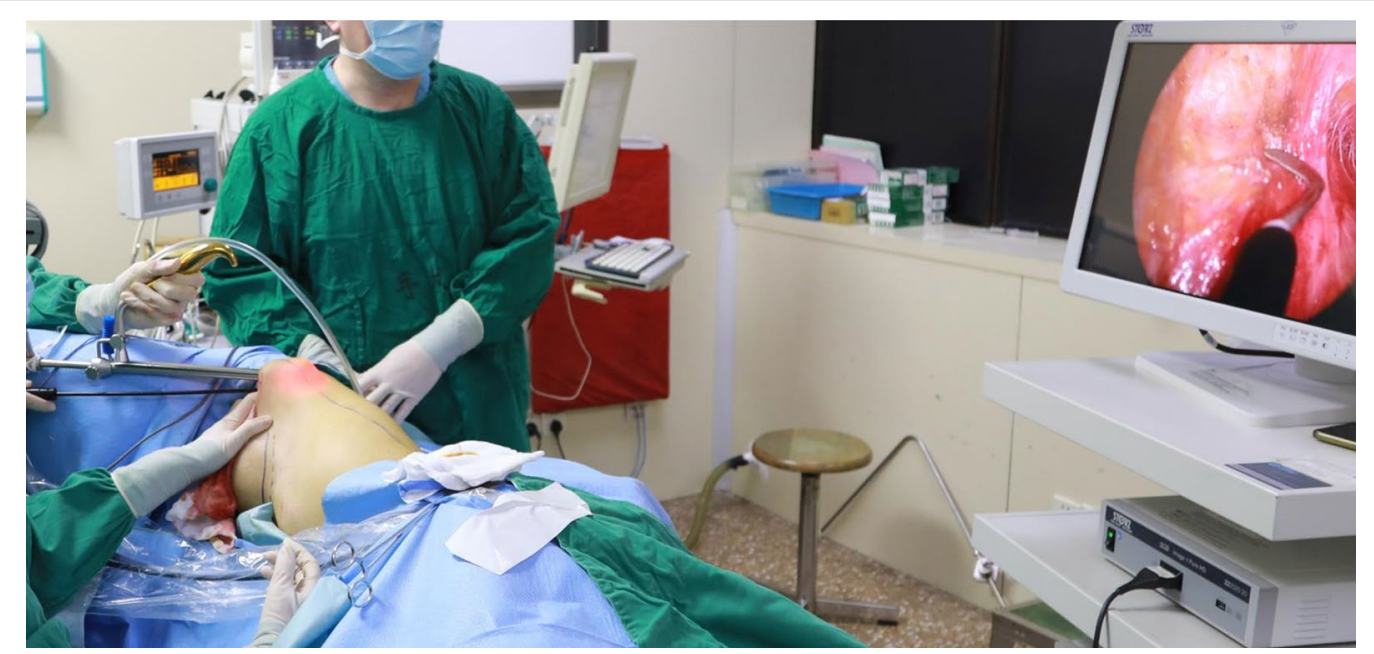

Fig. 2 Endoscopic approach to achieve the harvest of LDMF. The previous mastectomy incision extending to the posterior axillary fold is used as the approach to perform the endoscopy-assisted LDMF harvest. The subcutaneous plane is dissected first by a long monopolar electrosurgical hook. The endoscopic procedures are performed under the view of a video monitor. LDMF: latissimus dorsi muscle flap

Co., Ltd., Shanghai, China), then the dissection is carried out along the undersurface of the muscle. The endoscopic procedures are performed under the view of a video monitor. The thoracodorsal vessels are identified underneath the LDM with the aid of an L-shaped fiberoptic retractor (Changzhou Jinyang Medical Instrument Co., Ltd., Changzhou, China) under direct vision, and the pedicle is marked with a vessel loop which makes it easy to ensure its safety. When the dissection is completed on both the superficial and deep planes, an additional 4-cm-long transverse incision is applied on the posterior waist under the level of the posterior superior iliac ridge. The muscle is disinserted from the paravertebral origin through both the axillary and posterior lumbar incision under the endoscopic view, and the inferoposterior part dissection is also facilitated by the approach of the lumbar incision. The muscle then can be easily pulled out through the initial incision without tension and rotate to the recipient pocket temporarily. The donor site is checked and hemostasis is conducted with the aid 


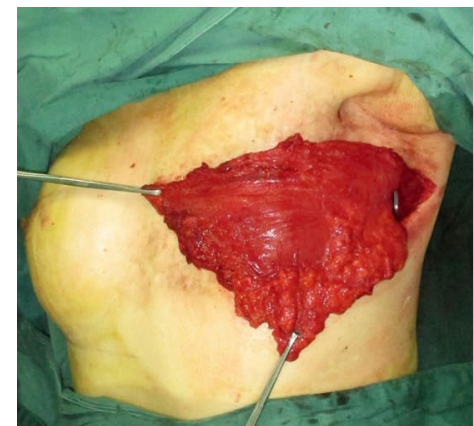

A

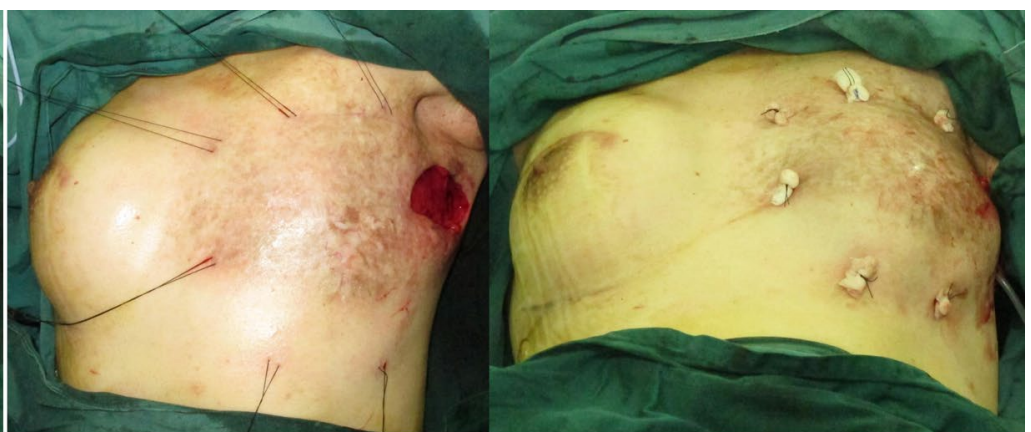

B

Fig. 3 The transfer of LDMF as an additional cover for the final implant. A LDMF is harvested using endoscopic technique through previous mastectomy incision approach and rotates to the affected chest wall. B The muscle flap is inserted into the expanded pocket, and the edges are fixed to the pocket through external bolster sutures, which serve as a complete cover for the implant. The bolster made of thirty-two layers of Vaseline gauze measuring $1.0 \mathrm{~cm}$ in length and $0.5 \mathrm{~cm}$ in width is secured by mattress suture of 0 silk suture. LDMF latissimus dorsi muscle flap

of endoscopy, and a closed suction drainage is applied through the lumbar incision followed by the closure of this incision.

The patient is shifted to the supine position. A capsulectomy is performed if necessary, and the pocket is irrigated with $60 \mathrm{ml}$ saline plus gentamicin $80,000 \mathrm{IU}$. The LDMF is inserted into the pocket, and the edges are fixed to the medial, inferior, and lateral aspects of the pocket through 4-6 external bolster sutures, which serve as a complete cover for the implant (Fig. 3). The bolster made of thirty-two layers of Vaseline gauze measuring $1.0 \mathrm{~cm}$ in length and $0.5 \mathrm{~cm}$ in width is secured by mattress suture of 0 silk suture $\left(\mathrm{ETHICON}^{\circledR}\right.$, Johnson \& Johnson Medical Ltd., Shanghai, China). Then the patient is kept in the sitting position, and a breast implant sizer $\left(\mathrm{MENTOR}^{\circledR}\right.$, Mentor Medical System B.V., Leiden, Netherlands) is routinely used to facilitate the determination of final implant size and location. A closed suction drainage is introduced in the implant pocket followed by the permanent implant (MENTOR ${ }^{\circledR}$, Mentor Medical System B.V., Leiden, Netherlands) placement under the LDMF. The skin incision is closed at last.

The drain tubes are removed when the output of each is less than $20 \mathrm{ml}$ per day postoperatively. Cotton pads with elastic bandages are applied for two weeks and an elastic vest is to be worn for two more weeks. Appropriate immobilization of the relevant shoulder joint in functional position for two weeks is recommended. Afterwards, the shoulder movement range should be restored gradually.

\section{Acquisition of the three-dimensional surface image}

Three-dimensional surface image (3D-SI) is acquired by VECTRA-XT stereophotogrammetry device (Canfield Scientific Inc, Fairfield, NJ, USA). Breast Sculptor ${ }^{\circledR}$ software (version 5.5.3, Canfield Scientific Inc, Fairfield, NJ, USA) is used to provide breast measurement data. When the breast boundaries are determined, the breast volume can be calculated automatically after the virtual chest wall is simulated from the landmarks by the software [5]. The 3D images of every patient were acquired at more than 6 months post-reconstruction follow-up visits.

\section{Evaluation of patient satisfaction}

An evaluation of patient reported outcomes was conducted by utilizing BREAST-Q Reconstruction Module Postoperative Scales Mandarin Version. The scales included Satisfaction with Breasts. The satisfaction was evaluated as follows [6]: excellent (score 81-100), the bilateral breasts were highly symmetrical, and the patient was highly satisfied; good (score 61-80), the reconstructed breast was a little asymmetrical with the contralateral side, and the patients was satisfied; fair (score 31-60), the reconstructed breast was asymmetrical with the contralateral breast, and the patient was not satisfied; and bad (score 0-30), the reconstructed breast presented distortion.

\section{Statistical analysis}

Breast volume asymmetry is assessed using the following formula: [right breast volume-left breast volume]/ the larger breast volume $\times 100 \%$, which represents the volume difference between the two breasts [7]. The data are analyzed using paired-sample $\mathrm{T}$ test. The measurement data are expressed as mean \pm standard deviation. $\mathrm{P}$ values are two-tailed, and $\mathrm{P}$ values $<0.05$ are considered significant. All the data are analyzed using IBM SPSS Statistics 25.0 (International Business Machines Corp., USA). 
Table 1 Demographic data of thirty-one patients undergoing endoscopy-assisted LDMF transfer combined with implant insertion after tissue expansion for breast reconstruction

\begin{tabular}{ll}
\hline Category $(\mathbf{n}=\mathbf{3 1})$ & Mean $\pm \mathbf{S D} / \mathbf{n}(\%)$ \\
\hline Age & $37.6 \pm 6.4$ \\
Body mass index $\left(\mathrm{kg} / \mathrm{m}^{2}\right)$ & $21.4 \pm 2.3$ \\
TNM anatomic stage (breast cancer) & \\
Stage I & $13(41.9 \%)$ \\
Stage II & $18(58.1 \%)$ \\
Final pathology & \\
Invasive ductal carcinoma & $24(77.4 \%)$ \\
Ductal carcinoma in situ & $7(22.6 \%)$ \\
Affected side & \\
Right & $13(41.9 \%)$ \\
Left & $18(58.1 \%)$ \\
Chemotherapy & \\
Yes & $28(90.3 \%)$ \\
No & $3(9.7 \%)$ \\
Radiation & \\
Yes & $3(9.7 \%)$ \\
No & $28(90.3 \%)$ \\
\hline
\end{tabular}

LDMF latissimus dorsi muscle flap

\section{Results}

\section{Patients}

Thirty-one patients were included in this research. The average age was 37.6years (26.0 to 50.0years), and the average body mass index (BMI) was $21.4 \mathrm{~kg} / \mathrm{m}^{2}(19.2$ to $29.7 \mathrm{~kg} / \mathrm{m}^{2}$ ). The TNM anatomic stages of breast cancer were I and II. The final pathological outcomes of the mastectomy specimens were invasive ductal carcinoma and ductal carcinoma in situ. Twenty-eight patients received chemotherapy. Two patients underwent radiation treatment at the end of tissue expansion and received LDMF transfer six months after the radiation, and one patient received radiation post-mastectomy and was applied delayed TE insertion. The demographic data are shown in Table 1.

\section{Reconstruction process}

Ten patients were treated with immediate TE placement, and twenty-one patients were provided with delayed TE insertion. The average time of LDMF harvest using the endoscopic technique was $90.4 \mathrm{~min}$ (70.0 to $120.0 \mathrm{~min}$ ). The average length of LDMF was $21.4 \mathrm{~cm}$ (18.0 to $27.0 \mathrm{~cm})$, and the width was $13.8 \mathrm{~cm}(12.0$ to $18.0 \mathrm{~cm})$. The mean volume of the final implant was $228.7 \mathrm{ml}$ $(175.0$ to $315.0 \mathrm{ml})$. The contralateral symmetry surgery included breast augmentation with an implant for six patients (three on the first stage and three on the second stage), breast augmentation with autologous fat grafting
Table 2 Clinical data during the reconstruction process

\begin{tabular}{lc}
\hline Category $(\mathbf{n}=\mathbf{3 1})$ & Mean $\pm \mathbf{S D} / \mathbf{n}(\%)$ \\
\hline Reconstruction timing & $10(32.3 \%)$ \\
Immediate & $21(67.7 \%)$ \\
Delayed & $90.4 \pm 13.7$ \\
LDMF harvest time (min) & $21.4 \pm 2.4$ \\
LDMF length (cm) & $13.8 \pm 1.4$ \\
LDMF width (cm) & $228.7 \pm 34.2$ \\
Final implant volume (ml) & \\
Contralateral symmetry surgery & $6(19.4 \%)$ \\
Augmentation with an implant & $4(12.9 \%)$ \\
Augmentation with fat grafting & $3(9.7 \%)$ \\
Mastopexy & $18(58.0 \%)$ \\
None &
\end{tabular}

LDMF latissimus dorsi muscle flap

for four patients (three on the first stage and one on the second stage), and mastopexy for three patients (one on the first stage and two on the second stage). The clinical data about the reconstruction process are shown in Table 2.

\section{Post-reconstruction}

The average drain time was 10.1 days (6 to 19 days). Four patients were found seroma formation, who were treated with aspiration. No other complications like hematoma, or wound healing abnormality were observed. All the patients got a follow-up from 6 months to 28 months (mean 11.2 months) post-reconstruction, and 3D images were acquired. Ten patients had a clinical capsular contraction with the Baker classification IB, sixteen patients with class II, five with class III, and no severe capsular contracture happened [8]. The average 3D-volume of the reconstructed breasts was $246.9 \mathrm{ml}(182.8$ to $327.8 \mathrm{ml})$, the average $3 \mathrm{D}$-volume of the contralateral breasts was $250.2 \mathrm{ml}(177.6$ to $340.2 \mathrm{ml}$ ), and the average breasts volume asymmetry post-reconstruction was $4.8 \%$ (2.3$12.3 \%)$. There was no significant difference between reconstructed and contralateral breasts $(P=0.256)$, which indicated that the bilateral breasts achieved good volume symmetry after reconstruction (Table 3). Twentynine patients finished the questionnaire of BREAST-Q at average 18 months post-reconstruction, and two patients did not complete it due to personal reasons. The average post-operative score of Satisfaction with Breasts was 69.1. Based on the evaluation of the BREAST-Q score, the outcome of Satisfaction with Breasts was excellent in five patients, good in twenty-two patients, and fair in two patients. Thus, the outcome was excellent or good in $87.1 \%$ of the cases (Table 4 ). Pre-/post-operative 
Table 3 Clinical data post-reconstruction

\begin{tabular}{lll}
\hline Category $(\mathbf{n}=\mathbf{3 1})$ & Mean $\pm \mathbf{S D} / \mathbf{n}(\%)$ & $\mathbf{P}$ \\
\hline Drain time (day) & $10.1 \pm 3.5$ & \\
Follow-up (month) & $11.2 \pm 4.9$ & \\
Complications & & \\
$\quad$ Seroma & $4(12.9 \%)$ & \\
$\quad$ None & $27(87.1 \%)$ & 0.256 \\
Volume of reconstructed breast $(\mathrm{ml})$ & $246.9 \pm 42.1$ & \\
Volume of contralateral breast $(\mathrm{ml})$ & $250.2 \pm 45.6$ & \\
Breasts asymmetry (\%) & $4.8 \pm 2.4$ & \\
\hline
\end{tabular}

$P$ paired-sample T test

Table 4 BREAST-Q reconstruction module Satisfaction with Breasts scale scores

\begin{tabular}{lll}
\hline Score & $\mathbf{n}(\%)$ & Mean \pm SD \\
\hline $81-100$ (Excellent) & $5(16.1 \%)$ & \\
$61-80$ (Good) & $22(70.9 \%)$ & \\
$31-60$ (Fair) & $2(6.5 \%)$ & \\
$0-30$ (Bad) & 0 & $69.1 \pm 10.3$ \\
None $^{\text {a }}$ & $2(6.5 \%)$ & \\
Average & &
\end{tabular}

The questionnaire was completed average 1.5 years after reconstruction

${ }^{a}$ Patients who did not complete the questionnaire due to personal reasons

photographs and 3D images of three patients are shown in Figs. 4, 5 and 6.

\section{Discussion}

Breast reconstruction is considered as a part of breast cancer treatment to restore breasts to their near-normal shape, size, and symmetry, and to enhance the quality of patients' lives. Nowadays, the demand for breast reconstruction is increasing constantly, which leads plastic surgeons to look for new methods to obtain a more natural and aesthetically pleasing appearance of the reconstructed breasts.

Because a large amount of skin and a considerable volume of inner tissue have been lost due to mastectomy, the restoration of skin envelope and the compensation for the breast tissue loss are two key issues that need to be considered during reconstructive surgery. In fact, tissue expansion two-stage reconstruction, which could expand the pectoralis major muscle and its overlapped skin at the same time, so as to restore the soft tissue cover, has become the most popular reconstruction method in China. Then volume replacement techniques, like implants insertion or distant autologous tissue flaps transfer, could help to compensate for the tissue loss. Among these, the implant is one of the most frequently used for most Chinese patients. Yet the implant always makes the breast feel stiff and the transition area between breast and chest wall looks unnatural, especially when the implant size is large to fit the large contralateral breast. As for the autologous tissue flap, mastectomy with LD flap transfer could only be beneficial for women with small-to-medium-sized breasts with upper outer quadrant breast cancer who desire breast conservation surgery $[3,4,9]$, because the provided volume is insufficient to achieve the total breast reconstruction.

In this study, we designed a new two-stage breast reconstruction protocol, which included tissue expansion on the first stage and implant insertion combined with LDMF transfer on the second stage. Using this method, the total extension of the pectoralis major muscle together with the transferred LDMF provide a robust muscular cover over the permanent implant, which makes the breast feel soft and smooth the transition region between the breast mound and the chest wall. Moreover, the additional volume of the LDM adding to the reconstructed breast allows choosing a small-sized implant, which also enhances the touch feeling of the breast.

Feng et al. [10] reported a similar two-stage breast reconstruction protocol, but the difference was that the LDMF transfer was applied together with the tissue expander insertion on the first stage. Regarding tumor staging, it is important to identify patients who will require adjuvant radiation therapy. Under this circumstance, the LDMF should be preserved as a salvage option for use in a delayed reconstruction setting. Because the non-irradiated autologous tissue flap could provide nonirradiated cellular elements which can lead to the repair of the dermal fibrosis resulted from post-mastectomy radiation therapy [11]. Moreover, it was also showed that the harvest of the LDMF is associated with a low complication rate and reliable results for delayed reconstruction of the irradiated breast [12, 13]. So, under consideration of the uncertainty about the application of radiation therapy in the immediate reconstruction cases, we leave the LDMF transfer on the second stage for breast reconstruction in this difficult to predict clinical scenario.

Previously, the breast envelope often needs to be enlarged with an LD skin paddle to maximize the size of the implant in a single-stage reconstruction. However, this leads to a skin patch presented on the reconstructed breast which does not match the color, texture, or thickness of the native breast skin [12]. In our new breast reconstruction protocol, tissue expansion and the use of endoscopic assistance in muscle harvest are operative modalities designed to avoid the cutaneous patch effect of the transposed musculocutaneous paddle, which results in no skin mismatch from the donor to the recipient site. 


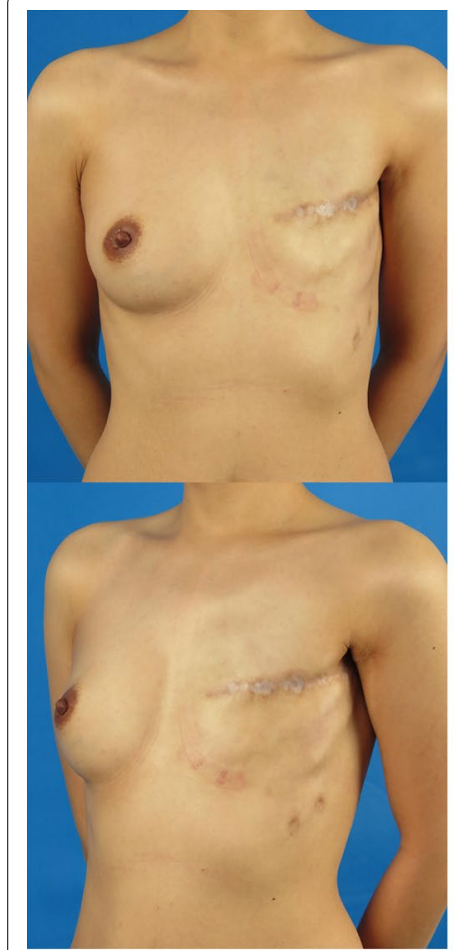

A

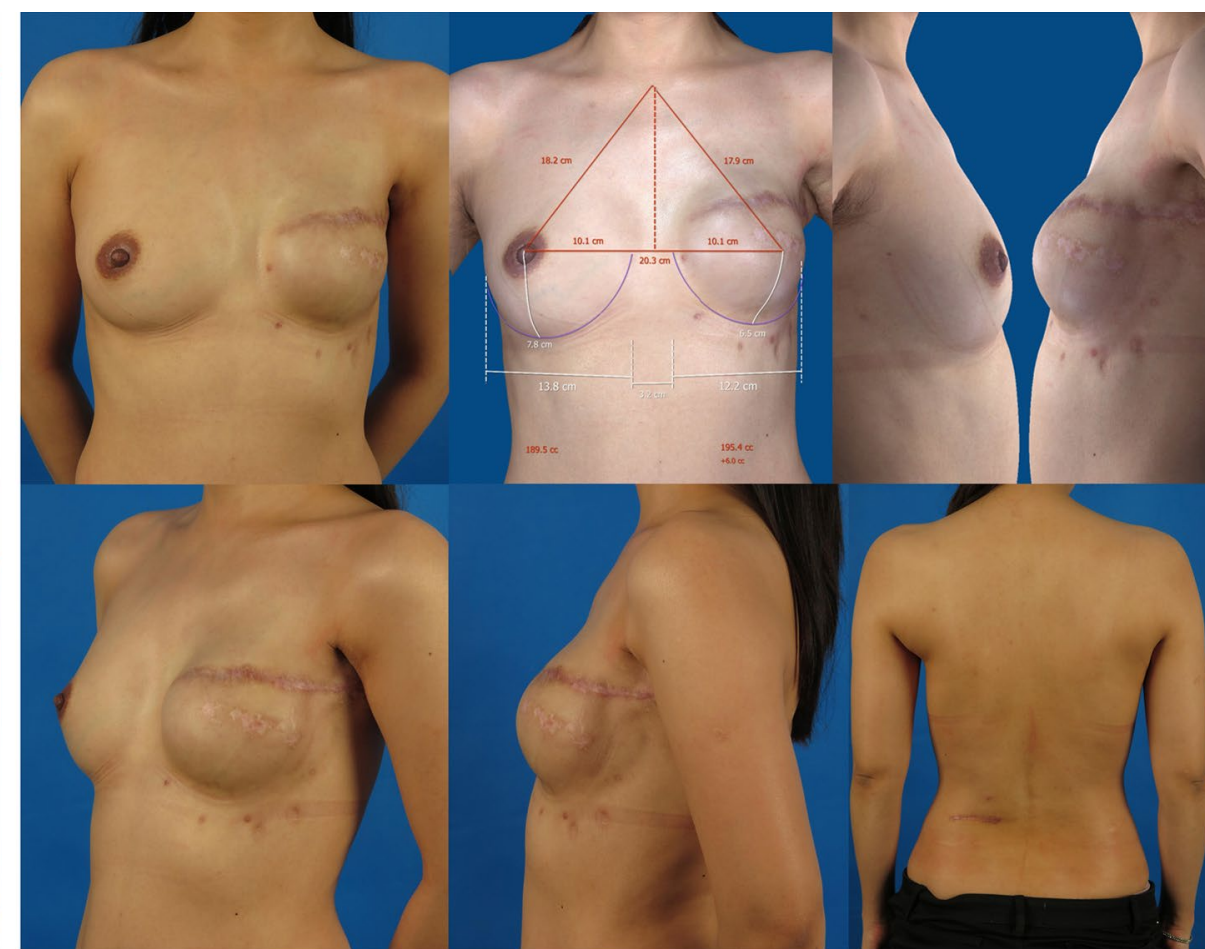

B

Fig. 4 The outcome of tissue expansion/implant with endoscopy-assisted LDMF transfer two-stage breast reconstruction at thirteen-month follow-up. A A 33 years old woman received left modified radical mastectomy because of breast cancer. The frontal and oblique pictures of the affected side were presented. B The patient underwent implant insertion with endoscopy-assisted LDMF transfer on the second stage of left breast reconstruction following tissue expansion on the first stage. The frontal, lateral and oblique pictures of the reconstructed breast were obtained at thirteen-month follow-up. The 3D image indicated the two breasts achieved good volume symmetry. The back view showed the scar of the lumbar incision was not obvious. LDMF latissimus dorsi muscle flap

The conventional LD flap harvest requires a long incision that often results in an apparent scar over the back which becomes one of the main concerns of the patients [9]. The endoscopy-assisted muscle harvest technique is becoming popular in breast reconstruction because it obviates the need for an obvious posterior donor site scar by using a small lateral extension of the mastectomy incision.

Many techniques for creating the optical cavity for endoscopic operation have been described already. Some authors preferred manual traction with endoscopic retractors [14-16] or operated with the aid of traction stitches in the skin [17], while others favored gas inflation using trocars to fit the laparoscopic instruments $[4,18]$ or robotic arms $[3,12,19,20]$. All these procedures achieved harvest of the LDMF with fewer visible scars than the conventional technique. Nevertheless, all of them created a vertical scar or three to four incisions vertically lined along the side of the chest for insertion of the trocars. Although a vertical incision is much easier to harvest the LDMF [16], a transverse incision is preferred for its better aesthetic outcome. Unlike setting the incision or multiple ports cut along the vertical posterior axillary line in other reports, we apply a transverse incision extending along the mastectomy scar, which enables the incision to be concealed under the axillary fold.

Dividing the LDM from its paravertebral origin and iliac attachments is the challenging part of the procedure, because of the narrow operative view and the difficulty in the resection of the distant LDM over the thorax anatomic curvature. Moreover, it is hard to control the bleeding during the medial dissection encountering the lumbar perforators, precisely where the access is most restricted. Some studies reported techniques for LDMF harvest via the mastectomy incision and axillary incision under the endoscopic guidance [14, 21]. Although these techniques have the advantage of no scar on the back, various specific retractors and other self-designed instruments are essential for the procedure. Moreover, these techniques require rich endoscopic surgery experience and a long learning curve to achieve efficiency and safety, which are quite unfriendly to beginners. 


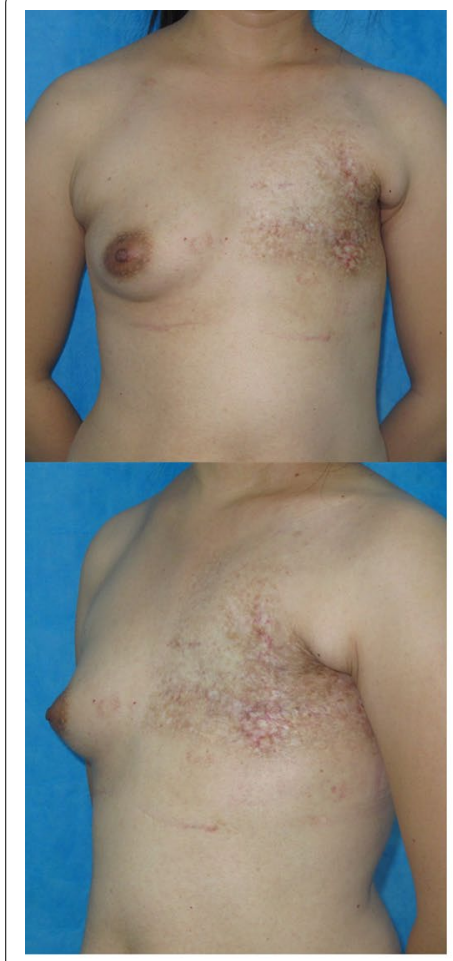

A

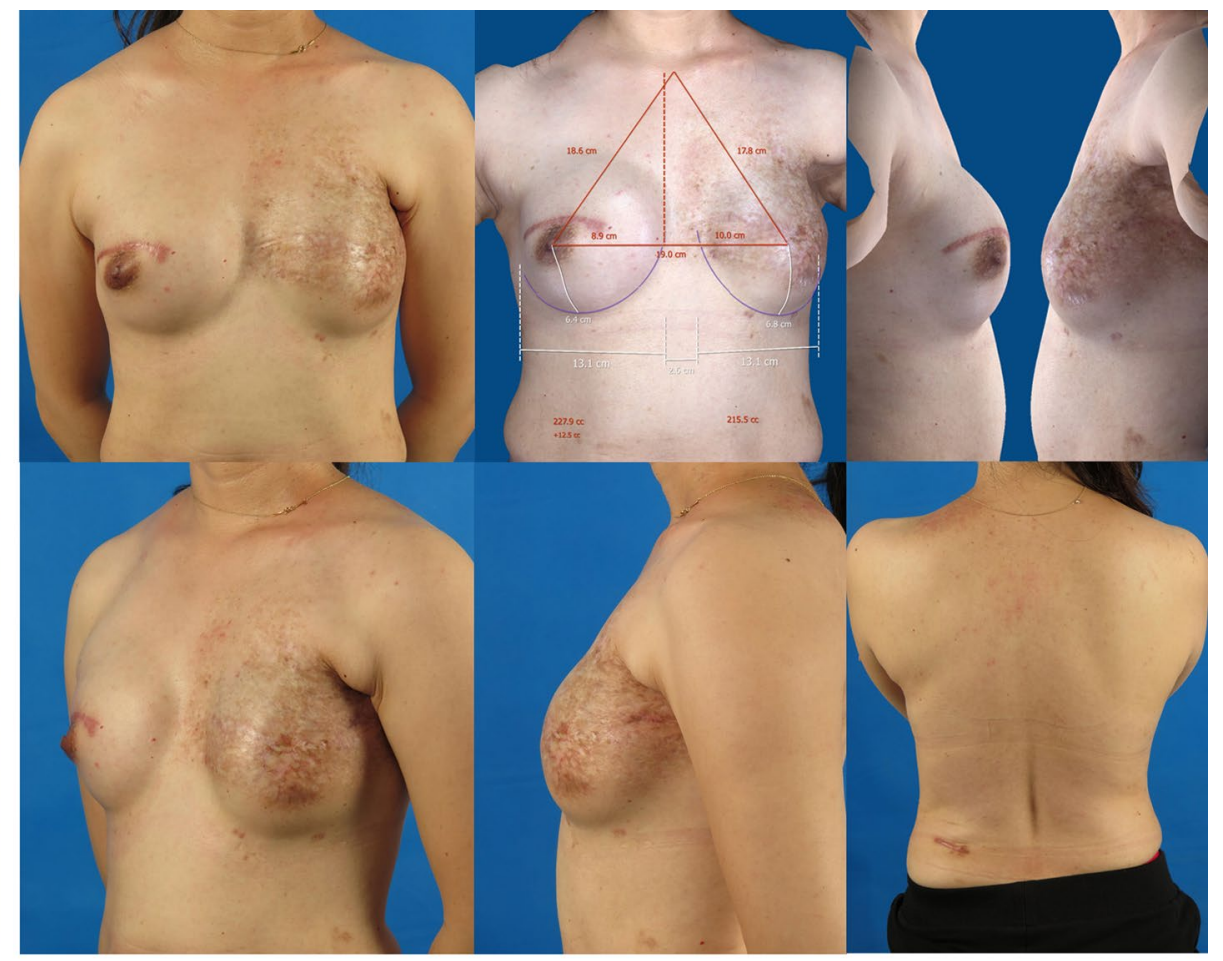

B

Fig. 5 The outcome of the patient who was applied tissue expansion/implant with endoscopy-assisted LDMF transfer two-stage left breast reconstruction combined with implant augmentation of right side at twelve-month follow-up. A A 33 years old woman underwent modified radical mastectomy for left breast cancer, then she received radiation therapy. The frontal and oblique pictures of the affected side were presented. B The patient received right nipple-sparing mastectomy because of fibroadenomatoid adenosis, followed by right breast augmentation with an implant, in the meanwhile, she was applied a tissue expander insertion on the first stage of left breast reconstruction. When the tissue expansion was finished, the patient underwent implant insertion with endoscopy-assisted LDMF transfer on the second stage of left breast reconstruction. The frontal, lateral and oblique pictures of the reconstructed breast were obtained at twelve-month follow-up. The 3D image indicated the two breasts achieved good volume symmetry. The back view showed the additional incision could be concealed when wearing pants. LDMF latissimus dorsi muscle flap

We add one more approach for endoscopy-assisted LDMF harvest to balance the difficulty of the procedure and the complication issues against the negative effects of longer scars. In this study, an additional short incision was applied on the posterior waist under the level of the posterior superior iliac ridge to facilitate the LDMF harvest, which made it easy to divide the inferoposterior origin of the muscle and perform the hemostasis. Also, this incision can be concealed perfectly when wearing pants.

The endoscopic technique does have a learning curve because of the lack of tactile sensation when using the long endoscopic instruments and the lack of depth nature from the two-dimensional video screen. Therefore, endoscopy-assisted LDMF harvest does require prolonged surgical time. Although various techniques had been employed to facilitate this procedure, the average LDMF harvest time was still around 120 240 min [4, 18, 22]. Even the latest da Vinci robotic technique required over 120 min $[19,23]$. The mean LDMF harvest time in this study was $90.4 \mathrm{~min}$, which was much shorter than previous studies. The short duration was attributed to the aid of the supplementary posterior lumbar approach. To be sure, the endoscopy-assisted harvest time can also decrease once the learning curve is overcome by the constant use of this technique.

Previous studies have demonstrated that this minimal invasive LDMF harvest technique can be performed with a lower complication rate compared with traditional open techniques [12]. And the most common complication of breast reconstruction with LD flap is donor site seroma [24]. There was $12.9 \%$ seroma occurrence rate postoperatively in this study, which is better than the $28.6 \%$ seroma formation rate in a research including 14 cases undergoing endoscopic LDMF harvest for breast reconstruction [4]. The good result might be attributed to the prolonged periods of drainage, elastic compression of the operation 


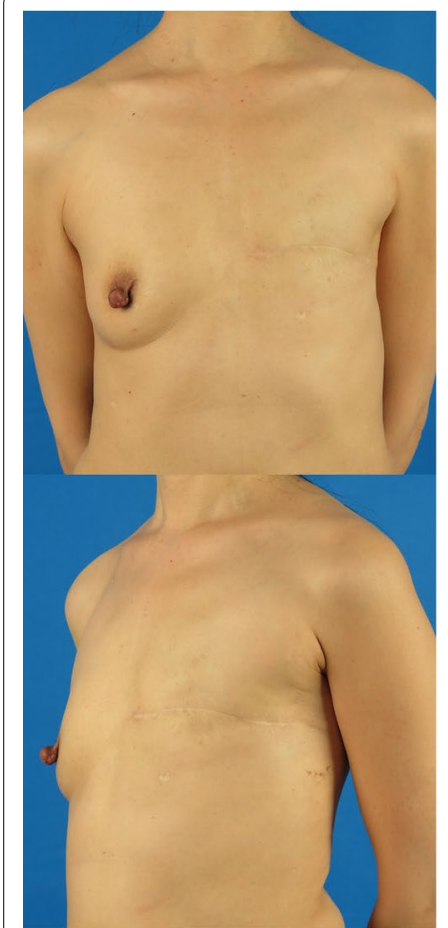

A

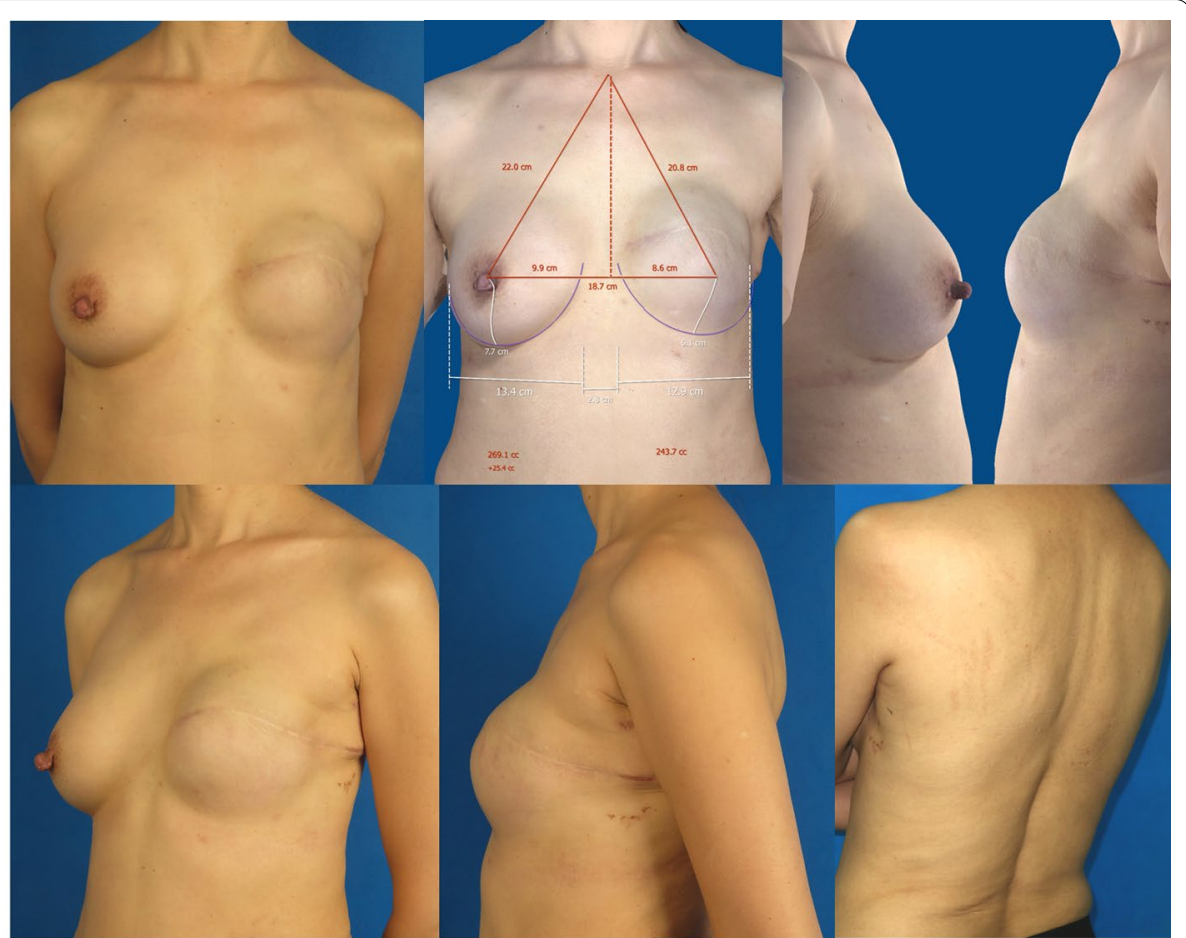

B

Fig. 6 The outcome of the patient who was applied tissue expansion/implant with endoscopy-assisted LDMF transfer two-stage left breast reconstruction combined with implant augmentation of right side at twenty-month follow-up. A A 50 years old woman underwent modified radical mastectomy for left breast cancer. The frontal and oblique pictures of the affected side were presented. $\mathbf{B}$ The patient underwent implant insertion with endoscopy-assisted LDMF transfer on the second stage of left breast reconstruction following tissue expansion on the first stage. In the meanwhile, she underwent right breast augmentation with an implant. The frontal, lateral and oblique pictures of the reconstructed breast were obtained at twenty-month follow-up. The 3D image indicated the two breasts achieved good volume symmetry. The back view showed the scar of the lumbar incision was almost invisible. LDMF latissimus dorsi muscle flap

areas, and immobilization of the shoulder joints. Compared with 8.0 days in a recent study applying endoscopyassisted LDMF harvest for breast reconstruction [21], the mean drain time in our research was 10.1 days. As for the reason, the strict criteria for drain removal was considered (less than $20 \mathrm{ml}$ VS $50 \mathrm{ml}$ per day). A longer time of drain tubes maintenance is prone to cause the relevant complications like secondary infection or wound dehiscence, so the meticulous medical care is essential.

There were several limitations in this study. First, the sample size of this research was small. Therefore, further studies involving a larger number of patients are needed to evaluate the effectiveness and safety of this type of breast reconstruction protocol. Second, although the mean follow-up time reached 11.2 months, it is not long enough to reveal unpleasant complications like capsular contracture which might occur many years after the surgery. Additionally, the final outcomes were evaluated only by the volume symmetry, while the contour symmetry including base width and projection are also important indicators to be assessed, which is believed to be involved in the subsequent researches. Despite the limitations, early success in this type of breast reconstruction protocol has indicated a good application prospect.

\section{Conclusions}

To the best of our knowledge, the novel type of twostage breast reconstruction protocol, which includes tissue expansion on the first stage and implant insertion with endoscopy-assisted LDMF transfer on the second stage, is reported for the first time in China. During the process, the use of endoscopic technique and a supplementary posterior lumbar approach allow the procedures to reduce visible scars, avoid the patch effect, while require short time for LDMF harvest and present low incidence of complications. The reported protocol is useful for breast reconstruction because it results in an objectively smaller scar at the donor site while achieves a good outcome of the reconstructed breast according to the evaluation of our patients. 


\section{Abbreviations}

LDMF: Latissimus dorsi muscle flap; TE: Tissue expander; 3D-SI: Three-dimensional surface image; BMI: Body mass index.

\section{Acknowledgements}

Not applicable.

\section{Authors' contributions}

Study concept and design: BL, YCX; Acquisition of data: JXM, YMS, YL; Data analysis: JXM, XC; Manuscript preparation: JXM; Study supervision: WTY, JZ. All authors read and approved the final manuscript.

\section{Funding}

This study was supported by a grant from Key Clinical Projects of Peking University Third Hospital (No. BYSY2017003).

\section{Availability of data and materials}

The datasets used and/or analysed during the current study are available from the corresponding author on reasonable request.

\section{Declarations}

\section{Ethics approval and consent to participate}

All methods were performed in accordance with the Declaration of Helsinki. The protocol used in this study was approved by Peking University Third Hospital Medical Science Research Ethics Committee (No. M2018278). Informed consent was obtained from all patients by written document.

\section{Consent for publication}

Informed consent for publication was obtained by written document from all patients whose photos were used in this manuscript.

\section{Competing interests}

The authors declare that they have no competing interests.

Received: 9 September 2021 Accepted: 30 December 2021

Published online: 08 January 2022

\section{References}

1. Huang NS, Quan CL, Ma LX, Si J, Chen JJ, Yang BL, Huang XY, Liu GY, Shen ZZ, Shao ZM, Wu J. Current status of breast reconstruction in China: an experience of 951 breast reconstructions from a single institute. Gland Surg. 2016;5(3):278-86.

2. Levine SM, Lester ME, Fontenot B, Allen RJ. Perforator flap breast reconstruction after unsatisfactory implant reconstruction. Ann Plast Surg. 2011;66(5):513-7.

3. Lai HW, Chen ST, Lin SL, Lin YL, Wu HK, Pai SH, Chen DR, Kuo SJ. Technique for single axillary incision robotic assisted quadrantectomy and immediate partial breast reconstruction with robotic latissimus dorsi flap harvest for breast cancer: a case report. Medicine (Baltimore). 2018;97(27):e11373.

4. Iglesias M, Gonzalez-Chapa DR. Endoscopic latissimus dorsi muscle flap for breast reconstruction after skin-sparing total mastectomy: report of 14 cases. Aesthetic Plast Surg. 2013;37(4):719-27.

5. Ma JX, Xia YC, Li B, Zhao HM, Lei YT. Unilateral tissue expander/implant two-stage breast reconstruction with the assistance of three-dimensional surface imaging. Aesthetic Plast Surg. 2020;44(1):60-9.

6. Zheng H, Zhu G, Guan Q, Fan W, Li X, Yu M, Xu J, Wu X. A retrospective study of latissimus dorsi flap in immediate breast reconstruction. Front Oncol. 2021;11:598604.

7. Tepper OM, Karp NS, Small K, Unger J, Rudolph L, Pritchard A, Choi M. Three-dimensional imaging provides valuable clinical data to aid in unilateral tissue expander-implant breast reconstruction. Breast J. 2008;14(6):543-50.

8. Spear SL, Baker JL Jr. Classification of capsular contracture after prosthetic breast reconstruction. Plast Reconstr Surg. 1995;96(5):1119-23.

9. Lee J, Jung JH, Kim WW, Park CS, Lee RK, Park HY. Endoscopy-assisted muscle-sparing Latissimus Dorsi muscle flap harvesting for partial breast reconstruction. BMC Surg. 2020;20(1):192.
10. Feng J, Pardoe Cl, Mota AM, Chui CH, Tan BK. Two-stage latissimus dorsi flap with implant for unilateral breast reconstruction: getting the size right. Archi Plast Surg. 2016;43(2):197-203.

11. Kronowitz SJ. Delayed-immediate breast reconstruction: technical and timing considerations. Plast Reconstr Surg. 2010;125(2):463-74.

12. Clemens MW, Kronowitz S, Selber JC. Robotic-assisted latissimus dorsi harvest in delayed-immediate breast reconstruction. Semin Plast Surg. 2014;28(1):20-5.

13. de Runz A, Boccara D, Bekara F, Chaouat M, Mimoun M. Outcome of 122 delayed breast reconstruction following post-mastectomy radiotherapy: the scarless latissimus dorsi flap with tissue expansion technique. Ann Chir Plast Esthet. 2017;62(1):23-30.

14. Yang CE, Roh TS, Yun IS, Kim YS, Lew DH. Immediate partial breast reconstruction with endoscopic latissimus dorsi muscle flap harvest. Arch Plast Surg. 2014;41(5):513-9.

15. Masuoka T, Fujikawa M, Yamamoto H, Ohyama T, Inoue Y, Takao T, Hosokawa K. Breast reconstruction after mastectomy without additional scarring: application of endoscopic latissimus dorsi muscle harvest. Ann Plast Surg. 1998;40(2):123-7.

16. Salibi A, Hart AM. Articulated endoscopic linear cutter stapler facilitates minimal access harvest of the latissimus dorsi muscle flap for chest wall reconstruction in Poland syndrome. Plast Reconstr Surg. 2014;134(5):856e-8e.

17. Serra-Renom JM, Serra-Mestre JM, Martinez L, D'Andrea F. Endoscopic reconstruction of partial mastectomy defects using latissimus dorsi muscle flap without causing scars on the back. Aesthetic Plast Surg 2013;37(5):941-9.

18. Xu S, Tang P, Chen $X$, Yang X, Pan Q, Gui Y, Chen L. Novel technique for laparoscopic harvesting of latissimus dorsi flap with prosthesis implantation for breast reconstruction: a preliminary study with 2 case reports. Medicine (Baltimore). 2016:95(46):e5428.

19. Selber JC, Baumann DP, Holsinger FC. Robotic latissimus dorsi muscle harvest: a case series. Plast Reconstr Surg. 2012;129(6):1305-12.

20. Ichihara S, Bodin F, Pedersen JC, Porto de Melo P, Garcia JC Jr, Facca S, Liverneaux PA. Robotically assisted harvest of the latissimus dorsi muscle: a cadaver feasibility study and clinical test case. Hand Surg Rehabil. 2016;35(2):81-4.

21. Liu C, Luan J, Ouyang Y, Zhuang Y, Xu B, Chen L, Li S, Fu S, Xin M. Breast reconstruction in Poland syndrome patients with latissimus dorsi myo flap and implant: an efficient endoscopic approach using single transverse axillary incision. Aesthetic Plast Surg. 2019;43(5):1186-94.

22. Missana MC, Pomel C. Endoscopic latissimus dorsi muscle flap harvesting. Am J Surg. 2007;194(2):164-9.

23. Chung JH, You HJ, Kim HS, Lee BI, Park SH, Yoon ES. A novel technique for robot assisted latissimus dorsi flap harvest. J Plast Reconstr Aesthet Surg. 2015;68(7):966-72.

24. Yezhelyev M, Duggal CS, Carlson GW, Losken A. Complications of latissimus dorsi flap breast reconstruction in overweight and obese patients. Ann Plast surg. 2013;70(5):557-62.

\section{Publisher's Note}

Springer Nature remains neutral with regard to jurisdictional claims in published maps and institutional affiliations.

Ready to submit your research? Choose BMC and benefit from

- fast, convenient online submission

- thorough peer review by experienced researchers in your field

- rapid publication on acceptance

- support for research data, including large and complex data types

- gold Open Access which fosters wider collaboration and increased citations

- maximum visibility for your research: over 100M website views per year

At BMC, research is always in progress.

Learn more biomedcentral.com/submissions 